\title{
Jacobi's Action and the Density of States
}

\section{J. David Brown}

Departments of Physics and Mathematics

North Carolina State University, Raleigh, NC 27695-8202

\section{James W. York, Jr.}

Department of Physics and Astronomy

University of North Carolina, Chapel Hill, NC 27599-3255

The authors have introduced recently a "microcanonical functional integral" which yields directly the density of states as a function of energy. The phase of the functional integral is Jacobi's action, the extrema of which are classical solutions at a given energy. This approach is general but is especially well suited to gravitating systems because for them the total energy can be fixed simply as a boundary condition on the gravitational field. In this paper, however, we ignore gravity and illustrate the use of Jacobi's action by computing the density of states for a nonrelativistic harmonic oscillator.

\section{DEDICATION}

We dedicate this paper to Dieter Brill in honor of his sixtieth birthday. His continued fruitful research in physics and his personal kindness make him a model colleague. JWY would especially like to thank him for countless instructive discussions and for his friendship over the past twenty-five years.

\section{INTRODUCTION}

Jacobi's form of the action principle involves variations at fixed energy, rather than the variations at fixed time used in Hamilton's principle. The fixed time interval in Hamilton's action becomes fixed inverse temperature in the "periodic imaginary time" formulation, thus transforming Hamilton's action into the appropriate (imaginary) phase for a periodic path in computing the canonical partition function from a Feynman functional integral (Feynman and Hibbs 1965). In contrast, fixed total energy is suitable for the microcanonical ensemble and, correspondingly, Jacobi's action is the phase in an expression for the density of states as a real-time "microcanonical functional integral" (MCFI) (Brown and York 1993b).

We wish to characterize briefly the canonical and microcanonical pictures. (We shall speak only of energy and (inverse) temperature here, ignoring the other possible conjugate pairs of variables in order to simplify the discussion.) In the canonical 
picture, with a fixed temperature shared by all constituents of a system, there are no constraints on the energy. This feature simplifies combinatorial (counting) problems for canonical systems and leads to the factorization of the partition function for weakly coupled constituents. For gravitating systems in equilibrium, the temperature is not spatially uniform because of gravitational red and blue-shift effects. In such cases the relevant temperature is that determined at the boundary of the system (York 1986). It can therefore be specified as a boundary condition on the metric (York 1986, Whiting and York 1988, Braden et al. 1990) and used in conjunction with Hamilton's principle, which is the form of the action for gravity in which the metric is fixed on the boundary (Brown and York 1992, 1993a). (The metric determines the lapse of proper time along the boundary.) On the other hand, equilibrium in the canonical picture is not always stable when gravity is present, as is well known. For some pertinent examples, see York (1986), Whiting and York (1988), and Braden et al. (1990).

With its constraint on the energy, the microcanonical picture leads to more robust stability properties. However, the energy constraint can complicate calculations of relevant statistical properties because the constituents of the system share from a common fixed pool of energy. For field theories, with a continuous infinity of degrees of freedom, the energy constraint restricts the entire phase space of the system unless gravity is taken into account. For gravitating systems, as a consequence of the equivalence principle, the total energy including that of matter fields is an integral of certain derivatives of the metric over a two-surface bounding the system. Therefore, if we specify as a boundary condition the energy per unit two-surface area, we have constrained the total energy simply by a boundary condition (Brown and York 1993a, 1993b). Thus, the canonical and microcanonical cases differ only in which of the conjugate variables (Brown et al. 1990), inverse temperature or energy, is specified on the boundary. The corresponding functional integrals, for partition function or density of states, differ in which action gives the correct phase, Hamilton's or Jacobi's.

We have recently applied this reasoning to the case of a stationary black hole (Brown et al. 1991a, 1991b, Brown and York 1993b). The MCFI, in a steepest descents approximation, shows that the logarithm of the density of states is one-quarter of the area of the event horizon (that is, the Bekenstein-Hawking entropy) (Brown and York 1993b). In the present paper we shall disregard gravity and obtain the density of states for a nonrelativistic harmonic oscillator. This is a relatively simple situation in which to recall the properties of Jacobi's action and to see the MCFI at work. 


\section{JACOBI'S ACTION}

Consider, for simplicity, a particle of mass $m$ with a one-dimensional configuration space. The Lagrangian form of Jacobi's action is (Lanczos 1970, Brown and York 1989)

$$
S_{E}[x]=\int d x \sqrt{2 m[E-V(x)]},
$$

where $V(x)$ is the potential energy and the energy $E$ is a fixed constant. $S_{E}[x]$ is extremized by varying the path freely except that the end points are fixed. Now introduce a parameter $\sigma$ increasing monotonically from $\sigma^{\prime}$ at one end of the path to $\sigma^{\prime \prime}$ at the other. Denoting $d x / d \sigma$ by $\dot{x}$, we can write the action as

$$
S_{E}[x]=\int_{\sigma^{\prime}}^{\sigma^{\prime \prime}} d \sigma \dot{x} \sqrt{2 m[E-V(x)]},
$$

where $x^{\prime}=x\left(\sigma^{\prime}\right)$ and $x^{\prime \prime}=x\left(\sigma^{\prime \prime}\right)$ are fixed. Jacobi's action is invariant under changes $\delta x$ induced by changes of parameterization that preserve the end-point values of $\sigma$.

For constructing the MCFI, we employ the canonical form of Jacobi's action. Because of the reparameterization invariance of $S_{E}[x]$, the corresponding canonical Hamiltonian $\dot{x}(\partial L / \partial \dot{x})-L$ vanishes identically. Furthermore, the canonical momentum

$$
p=\frac{\partial L}{\partial \dot{x}}=[2 m(E-V)]^{1 / 2}
$$

is independent of $\dot{x}$ in one dimension and, in general, does not allow one to solve for all the $\dot{x}$ 's as functions of the $p$ 's. Indeed, from (3) we obtain the "Hamiltonian constraint"

$$
\mathcal{H}(x, p) \equiv \frac{p^{2}}{2 m}+V(x)-E \approx 0 .
$$

Because the canonical Hamiltonian is zero, there are no secondary constraints and $\mathcal{H}$ is then trivially first class. Jacobi's action in canonical form is thus

$$
S_{E}[x, p, N]=\int_{\sigma^{\prime}}^{\sigma^{\prime \prime}} d \sigma[p \dot{x}-N \mathcal{H}(x, p)],
$$

where $N$ is a Lagrange multiplier. The equations of motion following from variation of (5) are

$$
\begin{aligned}
\dot{x} & =N[x, \mathcal{H}]=\frac{N p}{m} \\
\dot{p} & =N[p, \mathcal{H}]=-N \frac{\partial V}{\partial x} \\
\mathcal{H} & =\frac{p^{2}}{2 m}+V-E=0 .
\end{aligned}
$$


Combining (6) and (8) determines the multiplier as

$$
N=\dot{x}[2(E-V) / m]^{-1 / 2} .
$$

The interpretation of (9) is that

$$
d t=N d \sigma
$$

is the lapse of physical time, in accordance with the definition of energy.

The canonical statement of reparameterization invariance is that the action (5) is invariant under the gauge transformation given by

$$
\begin{aligned}
\delta x & =\epsilon[x, \mathcal{H}], \\
\delta p & =\epsilon[p, \mathcal{H}], \\
\delta N & =\dot{\epsilon},
\end{aligned}
$$

where $\epsilon\left(\sigma^{\prime}\right)=\epsilon\left(\sigma^{\prime \prime}\right)=0$. With the choice

$$
\epsilon(\sigma)=\left(\frac{\sigma-\sigma^{\prime}}{\sigma^{\prime \prime}-\sigma^{\prime}}\right) T-\int_{\sigma^{\prime}}^{\sigma} d \alpha N(\alpha),
$$

where $T$ is the total time

$$
T=\int_{\sigma^{\prime}}^{\sigma^{\prime \prime}} d \sigma N(\sigma)
$$

the lapse function is transformed to a constant, namely, $N=T /\left(\sigma^{\prime \prime}-\sigma^{\prime}\right)$. This shows that every history is gauge related to a history with a constant lapse, and the time $T$ is the gauge invariant part of the lapse function. If the histories under consideration are restricted to those with constant lapse, the gauge freedom of Jacobi's action is removed and (5) becomes

$$
S_{E}[x, p ; T)=\int_{\sigma^{\prime}}^{\sigma^{\prime \prime}} d \sigma\left[p \dot{x}-T \mathcal{H} /\left(\sigma^{\prime \prime}-\sigma^{\prime}\right)\right] .
$$

This form of Jacobi's action is a functional of $x(\sigma)$ and $p(\sigma)$ and an ordinary function of the time interval $T$. The classical equations of motion for (16), that is, the conditions for the extrema of (16), are given by (6) and (7) with $N=T /\left(\sigma^{\prime \prime}-\sigma^{\prime}\right)$ along with

$$
0=\frac{\partial S_{E}}{\partial T}=-\frac{1}{\left(\sigma^{\prime \prime}-\sigma^{\prime}\right)} \int_{\sigma^{\prime}}^{\sigma^{\prime \prime}} d \sigma \mathcal{H}
$$

Since (6) and (7) imply that $\mathcal{H}$ is constant, equations (6), (7), and (17) together imply $\mathcal{H}=0$. It follows that the form (16) for Jacobi's action is classically equivalent to (5), but has no gauge freedom. 


\section{FUNCTIONAL INTEGRAL FOR JACOBI'S ACTION}

The functional integral associated with Jacobi's action can be constructed by integrating over all histories $x(\sigma), p(\sigma), T$, with fixed endpoints $x\left(\sigma^{\prime}\right)=x^{\prime}$ and $x\left(\sigma^{\prime \prime}\right)=x^{\prime \prime}$, where the phase for each history is given by the action (16). Thus, the functional integral is

$$
Z_{E}\left(x^{\prime \prime}, x^{\prime}\right)=\frac{1}{2 \pi \hbar} \int d T \int_{x\left(\sigma^{\prime}\right)=x^{\prime}}^{x\left(\sigma^{\prime \prime}\right)=x^{\prime \prime}} \mathcal{D} x \mathcal{D} p \exp \left\{\frac{i}{\hbar} \int_{\sigma^{\prime}}^{\sigma^{\prime \prime}} d \sigma\left[p \dot{x}-T \mathcal{H} /\left(\sigma^{\prime \prime}-\sigma^{\prime}\right)\right]\right\}
$$

where $\mathcal{D} x \mathcal{D} p$ is (formally) the product over $\sigma$ of the Liouville phase space measure $d x(\sigma) d p(\sigma) /(2 \pi \hbar)$. The integration measure in (18) can be justified by appealing to a BRST analysis based on the canonical action (5), as is done in the Appendix of Brown and York (1993b).

The functional integral over $x(\sigma)$ and $p(\sigma)$ in (18) has the familiar form of the path integral associated with Hamilton's action, where $\sigma$ plays the role of time and the Hamiltonian is $T \mathcal{H} /\left(\sigma^{\prime \prime}-\sigma^{\prime}\right)$. This path integral can be written as the matrix elements of the evolution operator $\exp (-i T \hat{\mathcal{H}} / \hbar)$, so the path integral for Jacobi's action becomes

$$
Z_{E}\left(x^{\prime \prime}, x^{\prime}\right)=\frac{1}{2 \pi \hbar} \int d T<x^{\prime \prime}\left|e^{-i T \hat{\mathcal{H}} / \hbar}\right| x^{\prime}>.
$$

Hence, taking the integration of $T$ over all real values, we have

$$
Z_{E}\left(x^{\prime \prime}, x^{\prime}\right)=<x^{\prime \prime}|\delta(\hat{\mathcal{H}})| x^{\prime}>
$$

Note that $Z_{E}\left(x^{\prime \prime}, x^{\prime}\right)$ satisfies the time independent Schrödinger equation, namely $\hat{\mathcal{H}} Z_{E}\left(x^{\prime \prime}, x^{\prime}\right)=0$ (where $\hat{\mathcal{H}}$ acts on the argument $\left.x^{\prime \prime}\right)$, since formally $\hat{\mathcal{H}} \delta(\hat{\mathcal{H}})=0$.

From (20) it follows that the trace of $Z_{E}\left(x^{\prime \prime}, x^{\prime}\right)$ yields the density of states

$$
\nu(E)=\int d x Z_{E}(x, x)=\operatorname{Tr} \delta(\hat{\mathcal{H}})=\operatorname{Tr} \delta(E-\hat{H}),
$$

where $\hat{H}$ is the usual Hamiltonian operator. By combining this result with (18) we find that $\nu(E)$ can be written directly as a functional integral, the MCFI:

$$
\nu(E)=\frac{1}{2 \pi \hbar} \int d T \int \mathcal{D} x \mathcal{D} p \exp \left\{\frac{i}{\hbar} \int_{-\pi}^{\pi} d \sigma[p \dot{x}-T \mathcal{H} / 2 \pi]\right\} .
$$

For later convenience, the endpoint parameter values have been chosen to be $\sigma^{\prime}=-\pi$ and $\sigma^{\prime \prime}=\pi$. The derivation of the path integral (22) for the density of states shows 
that the integration can be described as a sum over all phase space curves that begin and end at some "base point" $x(\pi)=x(-\pi)=x$, plus an integral over the base point $x$. Then roughly speaking, the density of states is given by a sum over all periodic histories. However, to be precise, it should be recognized that the sum in (22) counts each closed phase space curve a continuous infinity of times because any point on the curve can serve as the base point $x$. Also observe that the integration in (22) is over all real values of the time interval $T$, rather than just positive values. This implies that the functional integral for $\nu(E)$ consists of a sum over pairs of histories with members contributing equal and opposite phases (Brown and York 1993b). As a consequence, the density of states so constructed is real.

\section{DENSITY OF STATES FOR THE HARMONIC OSCILLATOR}

We now turn to the evaluation of the MCFI (22) for the density of states of a simple harmonic oscillator with angular frequency $\omega$ and Hamiltonian constraint

$$
\mathcal{H}=\frac{p^{2}}{2 m}+\frac{m \omega^{2} x^{2}}{2}-E .
$$

The periodic nature of the histories suggests the use of Fourier series techniques (Feynman and Hibbs 1965) for this calculation. Accordingly, write the phase space coordinates as

$$
\begin{aligned}
& x(\sigma)=a_{0}+\sum_{k=1}^{\infty}\left(a_{k} \cos k \sigma+b_{k} \sin k \sigma\right), \\
& p(\sigma)=c_{0}+\sum_{k=1}^{\infty}\left(c_{k} \cos k \sigma+d_{k} \sin k \sigma\right) .
\end{aligned}
$$

The functional integral over $x(\sigma)$ and $p(\sigma)$ is replaced by a multiple integral over the coefficients in the Fourier series (24) and (25) with measure

$$
\mathcal{D} x \mathcal{D} p=J d a_{0} d c_{0} \prod_{k=1}^{\infty}\left(d a_{k} d b_{k} d c_{k} d d_{k}\right)
$$

Here, $J$ is (formally) the Jacobian of the transformation from $x(\sigma), p(\sigma)$ to $a_{0}, c_{0}$, $a_{k}, b_{k}, c_{k}, d_{k}$. The form (24), (25) of this transformation shows (again, formally) that $J$ should be a real constant, and should be independent of $T, m, \omega$, and $E$. ( $J$ should depend on $\hbar$, since $\hbar$ appears in the definition of $\mathcal{D} x \mathcal{D} p$.) One of the goals of the present calculation is to determine the real constant $J$ that characterizes the change of integration variables specified by (24) and (25). Note that by integrating freely over all Fourier coefficients $a_{0}, c_{0}, a_{k}, b_{k}, c_{k}, d_{k}$, we have each closed phase space curve correctly counted a continuous infinity of times. This is because the 
values of the Fourier coefficients depend on the choice of base point that is assigned the parameter value $\sigma=\pi$ (identified with $\sigma=-\pi$ ) on a given closed phase space curve.

With the change of variables (24), (25), the density of states (22) for the harmonic oscillator becomes

$$
\nu(E)=\frac{J}{2 \pi \hbar} \int d T \int d a_{0} d c_{0} \prod_{k=1}^{\infty}\left(d a_{k} d b_{k} d c_{k} d d_{k}\right) \exp \left\{i S_{E} / \hbar\right\},
$$

where the phase is obtained by substituting the Fourier series for $x(\sigma)$ and $p(\sigma)$ into the action (16):

$$
\begin{aligned}
S_{E} & =E T-\frac{m \omega^{2} T}{2} a_{0}^{2}-\frac{T}{2 m} c_{0}^{2} \\
& -\frac{1}{2} \sum_{k=1}^{\infty}\left\{2 \pi k\left(a_{k} d_{k}-b_{k} c_{k}\right)+\frac{T}{2 m}\left(c_{k}^{2}+d_{k}^{2}\right)+\frac{m \omega^{2} T}{2}\left(a_{k}^{2}+b_{k}^{2}\right)\right\} .
\end{aligned}
$$

The calculation is simplified by expanding $c_{k}$ and $d_{k}$ about the solutions to their "equations of motion". Accordingly, observe that the action (28) is extremized for $c_{k}$ and $d_{k}$ that satisfy

$$
\begin{aligned}
& 0=\frac{\partial S_{E}}{\partial c_{k}}=\pi k b_{k}-\frac{T}{2 m} c_{k}, \\
& 0=\frac{\partial S_{E}}{\partial d_{k}}=-\pi k a_{k}-\frac{T}{2 m} d_{k}
\end{aligned}
$$

Thus, define new integration variables $\bar{c}_{k}$ and $\bar{d}_{k}$ by

$$
\begin{aligned}
c_{k} & =\frac{2 \pi m}{T} k b_{k}+\bar{c}_{k}, \\
d_{k} & =-\frac{2 \pi m}{T} k a_{k}+\bar{d}_{k},
\end{aligned}
$$

and the action (28) becomes

$$
\begin{aligned}
S_{E} & =E T-\frac{m \omega^{2} T}{2} a_{0}^{2}-\frac{T}{2 m} c_{0}^{2} \\
& -\frac{1}{2} \sum_{k=1}^{\infty}\left\{\frac{T}{2 m}\left(\bar{c}_{k}^{2}+\bar{d}_{k}^{2}\right)+\frac{m}{2 T}\left(\omega^{2} T^{2}-4 \pi^{2} k^{2}\right)\left(a_{k}^{2}+b_{k}^{2}\right)\right\} .
\end{aligned}
$$

The integrations over $a_{0}, c_{0}, a_{k}, b_{k}, \bar{c}_{k}$, and $\bar{d}_{k}$ are now straightforward since these variables are uncoupled in the action (33). Moreover, for each value of $k$, the integrals over $\bar{c}_{k}$ are identical to the integrals over $\bar{d}_{k}$, and the integrals over $a_{k}$ are identical 
to the integrals over $b_{k}$. From these observations it follows that the density of states (27) can be written as

$$
\begin{aligned}
\nu(E) & =\frac{J}{2 \pi \hbar} \int d T d a_{0} d c_{0} \exp \left\{\frac{i}{\hbar}\left[E T-\frac{m \omega^{2} T}{2} a_{0}^{2}-\frac{T}{2 m} c_{0}^{2}\right]\right\} \\
& \times\left(\prod_{k=1}^{\infty} \int d a_{k} d \bar{c}_{k} \exp \left\{\frac{i}{\hbar}\left[\frac{m\left(4 \pi^{2} k^{2}-\omega^{2} T^{2}\right)}{4 T} a_{k}^{2}-\frac{T}{4 m} \bar{c}_{k}^{2}\right]\right\}\right)^{2} .
\end{aligned}
$$

Each of these integrals (excluding the integral over $T$ ) has the form of a Fresnel integral,

$$
\int d x \exp \left(i A x^{2}\right)=\sqrt{\frac{\pi}{|A|}} \exp (i \pi \operatorname{sign} A / 4),
$$

where the constant $A$ is real. In evaluating (34), it is helpful to note that the square of the Fresnel integral (35) is $i \pi / A$. The result is

$$
\nu(E)=-i J \int d T \frac{1}{\omega T} \prod_{k=1}^{\infty}\left[\left(\frac{2 \hbar}{k}\right)^{2}\left(1-\frac{\omega^{2} T^{2}}{4 \pi^{2} k^{2}}\right)^{-1}\right] \exp \left\{\frac{i}{\hbar} E T\right\} .
$$

Now use the identity

$$
\sin x=x \prod_{k=1}^{\infty}\left(1-\frac{x^{2}}{\pi^{2} k^{2}}\right)
$$

to obtain

$$
\nu(E)=\frac{-i J}{2} \prod_{k=1}^{\infty}\left(\frac{2 \hbar}{k}\right)^{2} \int d T \frac{1}{\sin (\omega T / 2)} \exp \left\{\frac{i}{\hbar} E T\right\} .
$$

Next, express the inverse of $\sin (\omega T / 2)$ as

$$
\begin{aligned}
\frac{1}{\sin (\omega T / 2)} & =\frac{2 i}{e^{i \omega T / 2}-e^{-i \omega T / 2}}=2 i e^{-i \omega T / 2} \frac{1}{1-e^{-i \omega T}} \\
& =2 i e^{-i \omega T / 2} \sum_{n=0}^{\infty} e^{-i \omega T n}
\end{aligned}
$$

and insert this result into (38). Integrating the series term-by-term, we obtain

$$
\nu(E)=2 \pi \hbar J \prod_{k=1}^{\infty}\left(\frac{2 \hbar}{k}\right)^{2} \sum_{n=0}^{\infty} \delta(E-\hbar \omega(n+1 / 2)) .
$$

This result shows that the Jacobian $J$ for the change of variables (24), (25) should be identified with the (real, infinite) constant

$$
J=\frac{1}{2 \pi \hbar} \prod_{k=1}^{\infty}\left(\frac{k}{2 \hbar}\right)^{2}
$$


Then the density of states becomes

$$
\nu(E)=\sum_{n=0}^{\infty} \delta(E-\hbar \omega(n+1 / 2)),
$$

which is the anticipated result showing that for the harmonic oscillator $\nu(E)$ is a sum of delta functions peaked at half-odd-integer multiples of $\hbar \omega$.

Finally, we note that the various quantum-statistical and thermodynamical properties of a system can be obtained from its density of states. In particular, the canonical partition function $Z(\beta)$ is defined as the Laplace transform of $\nu(E)$, and from $Z(\beta)$ the heat capacity, entropy, and other thermodynamical quantities can be found. For the harmonic oscillator with density of states (42), the partition function is

$$
Z(\beta)=\int_{0}^{\infty} d E \nu(E) e^{-\beta E}=\sum_{n=0}^{\infty} e^{-\beta \omega \hbar(n+1 / 2)}
$$

which is the well known result.

\section{ACKNOWLEDGMENTS}

This research was supported by National Science Foundation grant PHY-8908741.

\section{REFERENCES}

Braden, H. W., J. D. Brown, B. F. Whiting, and J. W. York (1990). Charged black hole in a grand canonical ensemble. Physical Review, D42, 3376-3385.

Brown, J. D. and J. W. York (1989). Jacobi's action and the recovery of time in general relativity. Physical Review, D40, 3312-3318.

Brown, J. D., G. L. Comer, E. A. Martinez, J. Melmed, B. F. Whiting, and J. W. York (1990). Thermodynamic ensembles and gravitation. Classical and Quantum Gravity, 7, 1433-1444.

Brown, J. D., E. A. Martinez, and J. W. York (1991a). Rotating black holes, complex geometry, and thermodynamics. In Nonlinear Problems in Relativity and Cosmology, Eds J. R. Buchler, S. L. Detweiler, and J. R. Ipser. New York Academy of Sciences, New York.

Brown, J. D., E. A. Martinez, and J. W. York (1991b). Complex Kerr-Newman geometry and black hole thermodynamics. Physical Review Letters, 66, 22812284 . 
Brown, J. D. and J. W. York (1992). Quasi-local energy in general relativity. In Mathematical Aspects of Classical Field Theory, Eds M. J. Gotay, J. E. Marsden, and V. E. Moncrief. American Mathematical Society, Providence.

Brown, J. D. and J. W. York (1993a). Quasilocal energy and conserved charges derived from the gravitational action. To appear in Physical Review, D47.

Brown, J. D. and J. W. York (1993b). Microcanonical functional integral for the gravitational field. To appear in Physical Review, D47.

Feynman, R. P. and A. R. Hibbs (1965). Quantum Mechanics and Path Integrals. McGraw Hill, New York.

Lanczos, C. (1970). The Variational Principles of Mechanics. University of Toronto Press, Toronto.

Whiting, B. F. and J. W. York (1988). Action principle and partition function for the gravitational field in black-hole topologies. Physical Review Letters, 61, 13361339 .

York, J. W. (1986). Black-hole thermodynamics and the Euclidean Einstein action. Physical Review, D33, 2092-2099. 